\title{
Globular cluster system erosion in elliptical galaxies
}

\author{
R. Capuzzo-Dolcetta and A. Mastrobuono-Battisti
}

Dep. of Physics, Sapienza, University of Roma, P.le A. Moro 5, 00185, Roma, Italy

e-mail: [roberto.capuzzodolcetta; alessandra.mastrobuonobattisti]@uniroma1.it

Received 2 April 2009 / Accepted 5 August 2009

\begin{abstract}
Context. We analyse data of 8 elliptical galaxies to study the difference between the radial distributions of their globular cluster systems (GCSs) and their galactic stellar component. In all galaxies studied, the GCS density profile is significantly flatter towards the galactic centre than that of the stars.

Aims. A flatter profile of the radial distribution of the GCS with respect to that of the galactic stellar component is a difference with astrophysical relevance. A quantitative comparative analysis of the profiles may provide insight into both galaxy and globular cluster formation and evolution. If the difference is caused by erosion of the GCS, the missing GCs in the galactic central region may have merged around the galactic centre and formed, or at least increased in mass, the galactic nucleus. Observational support to this are the correlations between the galaxy integrated absolute magnitude and the number of globular clusters lost and that between the central massive black hole mass and the total mass of globular clusters lost.

Methods. We fitted both the stellar and globular cluster system radial profiles of a set of galaxies observed at high resolution. We found that the GCS profile is less sharply peaked at the galactic centre than the stellar one. Assuming that this difference is caused by GCS evolution, starting from a radial distribution initially indistinguishable from that of stars, we can evaluate by a simple normalization procedure the number (and mass) of GCs that have "disappeared".

Results. The number of missing globular clusters is significant, ranging from $21 \%$ to $71 \%$ of their initial population abundance in the eight galaxies examined. The corresponding mass lost to the central galactic region is (for every galaxy of the sample) in the $2.77 \times 10^{7}-1.58 \times 10^{9} M_{\odot}$ interval. All the transported mass towards the central galactic regions have had probably an important effect on the innermost galactic zone, including its violent transient activity (AGN) and local massive black hole formation and growth.
\end{abstract}

Key words. galaxies: general - galaxies: elliptical and lenticular, cD - galaxies: evolution - galaxies: star clusters - galaxies: nuclei

\section{Introduction}

Many elliptical galaxies contain quite populous globular cluster systems (hereafter GCSs), that are, usually, less concentrated towards the galactic centre than the bulge-halo stars. Since the seminal review by Harris \& Racine (1979) an enormous amount of work has been devoted to GCS identification in external galaxies and study of their properties. Regarding the GCS radial profiles, we recall, for the sake of example, recent papers of Bassino et al. (2006), Goudfrooij et al. (2007), Lee et al. (2008), Rhode et al. (2007), Sikkema et al. (2006), Spitler et al. (2006), Spitler et al. (2008), Rhode \& Zepf (2003), Peng et al. (2004) and Harris et al. (2009).

Although this characteristic may not be common to all galaxies, no case, at present, is known where the halo stars are less concentrated than the GCS. There is also general agreement that the difference between the two radial distributions is real and not caused by a selective bias. Consequently, different hypotheses have been proposed to explain this feature. Among these, two seem the most probable. The first hypothesis is that the difference between the two distributions reflects the different formation ages of the two systems, as suggested by Harris \& Racine (1979) and Racine (1991); in their opinion globular clusters formed earlier, when the galaxy mass density distribution was less centrally peaked. Also Forbes et al. (1996) and Ashman \& Zepf (1998), on an observational basis, and
Bekki \& Forbes (2006), on a theoretical basis, suggest that the halo-bulge and the globular cluster components may have been formed independently and thus their profiles should not, initially, be identical. Hydrodynamical simulations performed by Hopkins et al. (2009) suggest, for cuspy ellipticals, that the centre of these objects have been constructed by gaseous dissipation in mergers of gas-rich progenitors. The pre-existing stars (and clusters) should not participate in this dissipation, and instead be transported to greater radii. In this scheme, the merged system would not have identical cluster and stellar distributions.

However, these hypotheses have problems in explaining why the stellar and GCS distributions are usually similar in the outer galactic regions. Of course, there are a few exceptions such as the two giant ellipticals NGC 4472 (M 49) and NGC 4486 (M 87), and the lenticular galaxy NGC 4374 in the Virgo cluster, whose GCS distribution seems to be a more radially extended than the stellar component, while the lenticular galaxy NGC 4406, also in Virgo and close to NGC 4374, has a more steeply declining slope of the GCS radial profile than that of the galactic stellar component. These substantially different behaviours can both be attributed to tidal effects, in the sense that in some galaxies an external perturber may cause a tidal stripping of GCs in the outskirts causing depletion of the outer radial population with a corresponding enrichment of the GCS of the perturbing (likely massive) galaxy. This scheme is sketched in Elmegreen (1999) and has a support by numerical simulations 
of Forte et al. (1982) and Muzzio (1987). In any case, all of these bright galaxies are located at the centre of the cluster potential well, and their outskirts are noticeably distorted, because of gravitational interactions with other Virgo cluster galaxies and because of material acquired during cannibalistic encounters, so they cannot be considered as representative of the "average" elliptical.

Another explanation of the difference between the inner GCS and galactic stellar component radial profiles is based on the simpler assumption of a coeval birth of globular clusters and halo stars and additional evolution of the GCS radial distribution, while the collisionless halo remains almost unchanged.

The GCS evolution is caused by dynamical friction, which causes massive clusters to move very close to the galactic centre, where they are tidally disrupted by interactions with the growing nucleus, as first proposed by Tremaine et al. (1975) for M 31. This view of the galactic nuclei formation was, later, abandoned because approximated evaluations of the dynamical friction time scale for GCs to be confined around the galactic center indicated it was too long to account for a rapid accretion of mass of the central galactic regions (see for instance Fall \& Rees 1977 and Lauer \& Kormendy 1986, the latter authors referring to M 87). This view has been shown to be erroneous, because it is based on the oversimplified assumption that GCs move on quasi-circular orbits at large distances from the galactic center, where the star density is low and, consequently, so is the dynamical friction braking of even massive galactic satellites. A, more probable, radially biased orbital distribution for GCs significantly shortens the dynamical friction timescale. Additionally, the efficiency of dynamical friction is higher in triaxial galaxies, where box orbits maximize the effect of dynamical friction, as first suggested by Ostriker (1988). We note that box orbits represent a significant fraction of orbits in stable triaxial galaxies; the prototype of selfconsistent triaxial models, which was studied by Schwarzschild (1979) by means of his orbital superposition technique, has axial ratios 1:0.625:0.5, contains a large fraction of box orbits, and is stable, as demonstrated by Smith \& Miller (1982). The density concentration on and near the long axis of a triaxial figure is provided by the major family of box orbits which thus support the deviation from axial symmetry (Vietri \& Schwarzschild 1983).

Also the tidal interaction with a compact nucleus is enhanced in a triaxial potential (Ostriker et al. 1989; Capuzzo-Dolcetta \& Tesseri 1997; Capuzzo-Dolcetta \& Tesseri 1999; Capuzzo-Dolcetta \& Vicari 2005). The combined effects of these dynamical mechanisms act to deplete the GCSs in the central, denser, galactic regions, leaving the outer profile unaltered and similar to the profile of the halo stars; alternatively, when the galaxy interacts significantly with other galaxies, the GCS may radially expand towards outer regions. Based on the hypothesis that the initial GCS and halo-bulge radial distributions were similar, an accurate analysis of the observations would allow us to estimate the number of "missing clusters" and therefore the mass removed from the GCSs.

Two objections are often made against the evolutionary scheme. One is that in the hypothesis of efficient dynamical friction braking, which acts differentially on clusters of different mass, one expects to detect a radial dependence of the GC luminosity function, which is not usually observed. The radial dependence may not necessarily be expected because the two main external effects on the GCSs, dynamical friction and tidal disruption, affect different ends of the mass (luminosity) function leaving the average value almost unaltered (Capuzzo-Dolcetta \& Tesseri 1997). Another objection is that the observed, usually high, metallicities of the central galactic regions are incompatible with the, usually low, metal content of GCs. This objection relies on the presently observed GC population being representative of the initial one, which in its massive $\left(M>10^{6} M_{\odot}\right)$ tail should be lost to the inner galactic zone by strong braking. If the well known mass-metallicity relation indeed extends to very massive globular cluster scale, it is no surprise that massive, orbitally decayed GCs are also metal rich.

McLaughlin (1995), Capuzzo-Dolcetta \& Vignola (1997), Capuzzo-Dolcetta \& Tesseri (1999) and Capuzzo-Dolcetta \& Donnarumma (2001), estimated, by scaling the radial surface profiles of the halo stars of a galaxy to that of its GCS, the number of missing globular clusters to be the integral of the difference between the two radial profiles. Capuzzo-Dolcetta \& Vignola (1997) and Capuzzo-Dolcetta \& Tesseri (1999) suggested that the compact nuclei of the Galaxy, M 31 and M 87, as well as those in many other galaxies, may have accreted many decayed globular clusters in the first few Gyr of their life.

In this paper we extend the comparison of the GCS and star distribution in galaxies, studying eight galaxies for which good photometric data are available in the literature to draw reliable radial profiles.

In Sect. 2 we summarize the way, discussed deeply in previously mentioned papers, to derive, from the comparison of the GCS-stars radial profiles in a galaxy, the number and mass of GCs that have disappeared due to the time evolution of the GCS; in Sect. 3, we present and discuss the observational data, as well as the analytical fit expressions to the density profiles; in Sect. 4, we present the extension, to the data of this work, of the correlation found by Capuzzo-Dolcetta \& Donnarumma (2001) between the mass lost by the GCS, the host galaxy luminosity and the mass of the galactic central massive black hole. Finally, in Sect. 5 we summarize our results and draw some general conclusions. An error analysis of the methods used is presented in Appendix A.

\section{The estimate of both the number and total mass of globular clusters lost}

Based on the hypothesis that the flattening of the GCS distribution in the central regions, compared to the distribution of the stars in the galactic bulge, is caused by the evolution of the GCS, the number of GCs that are accreted inward to the centre of the galaxy is inferred by calculating the simple difference of the (normalized) density profiles integrated over the entire radial range (see McLaughlin 1995). A general discussion of the problems in evaluating the number and mass of missing clusters in this way can be found in Capuzzo-Dolcetta \& Vignola (1997), Capuzzo-Dolcetta \& Tesseri (1999), and Capuzzo-Dolcetta \& Donnarumma (2001), and is not repeated here.

In all cases, apart from NGC 4472, we assume as a fitting function for the GCS projected radial distribution in the form of a "modified core model", i.e., a law

$\Sigma_{\mathrm{GC}}(r)=\frac{\Sigma_{0}}{\left[1+\left(r / r_{\mathrm{c}}\right)^{2}\right]^{\gamma}}$

where $\Sigma_{0}, r_{\mathrm{c}}$, and $\gamma$ are free parameters. The choice of this function to fit the GCS profiles is motivated by the good agreement found with almost all the data used in this paper, as confirmed by, both, the local maximum deviation of the fitting formula from the observed data and the computed $\chi^{2}$.

For the galaxy stellar profile, $\Sigma_{\mathrm{s}}(r)$, we rely on the fitting formulas provided by the authors of the various papers from which 
we derived the data, which may change case by case, depending on how good the approximations are for observed data. The choice of taking outer radial galaxy stellar profiles from the same source paper from which we derived the data for the GCS profile was made for the sake of homogeneity with the GCS profiles because the galaxy photometry was usually taken with the same instrument, in the same photometric band, and, probably, in comparable observation conditions. We note that this was possible for the external radial profiles of NGC 1400, NGC 1407, NGC 4374, and NGC 4636 (the inner distribution was assumed to be core-like), while for NGC 3258 and NGC 3268 a single law (modified Hubble's) was adopted across the entire radial extension. In the two remaining cases, NGC 4406 and NGC 4472, we selected the most reliable and recent among the various photometric profiles found in the literature.

A check of the sensitivity to the choice of galactic photometry is found in the discussion of NGC 4636 data (Sect. 3.8). The "initial" distribution of GCs, $\Sigma_{\mathrm{GC}, 0}(r)$, is assumed to be equal in shape to the present stellar profile; consequently, it can be obtained by a vertical translation of the stellar profile, $\Sigma_{\mathrm{s}}(r)$, to match the present GCS distribution $\left(\Sigma_{\mathrm{GC}}(r)\right.$ as given by Eq. (1)) in the outer zone. We calculate the number of missing (lost) clusters to be the surface integral of the difference between $\Sigma_{\mathrm{GC}, 0}(r)$ and $\Sigma_{\mathrm{GC}}(r)$ over the radial range $\left[0, r_{\mathrm{max}}\right]$ where these two profiles differ, where $r_{\max }$ is defined to be the radial coordinate of the intersection of the GCS and stellar radial profiles that minimizes, after a vertical shift of the stellar radial profile, the squared of the difference between the GCS and stellar density distributions over the range $\left[r_{\max }, R\right] . R$ is the largest radial distance covered by the observations (for the values of $R$, we rely on the papers from which we derived the GC distribution data). Consequently, the number of lost clusters is

$N_{1}=2 \pi \int_{0}^{r_{\max }}\left[\Sigma_{\mathrm{GC}, 0}(r)-\Sigma_{\mathrm{GC}}(r)\right] r \mathrm{~d} r$.

The present number of GCs, $N$, is obtained by integrating $\Sigma_{\mathrm{GC}}(r)$ over the entire radial range, $[0, R]$. The values of $N$ obtained with this method usually differ from those given by the authors of the papers, but for the purposes of this paper what is important is the difference between $N$ and $N_{1}$ and, so, it is crucial to have a homogeneous way of determining them.

The initial number of GCs in a galaxy is, indeed, estimated to be $N_{\mathrm{i}}=N_{1}+N$.

The numerical values of $N_{\mathrm{l}}, N_{\mathrm{i}}$, and $N$ are functions of both the fitting parameters and the integration limits. These dependences and their contribution to the errors in the final results are discussed in Appendix A.

An estimate of the mass removed from the GCS $\left(M_{1}\right)$ can be obtained from the number of GCs lost, $N_{1}$, and the estimate of the mean mass of the missing globular clusters, $\left\langle m_{1}\right\rangle$. A priori, the determination of $\left\langle m_{1}\right\rangle$ require the knowledge of the initial mass spectrum of the CGS, that has experienced an evolutionary erosion. However, the most relevant evolutionary phenomena (tidal shocking and dynamical friction) act on opposite sides of the initial mass function, and so we expect that the mean value of the globular cluster mass has not changed very much in time (see Capuzzo-Dolcetta \& Tesseri 1997 and Capuzzo-Dolcetta \& Donnarumma 2001). Hence, we can assume the present mean value of the mass of globular clusters, $\langle m\rangle$, to be a good reference value for $\left\langle m_{1}\right\rangle$.

For NGC 4374, NGC 4406, and NGC 4636, we calculated $\langle m\rangle$ using their GC luminosity functions (GCLFs) and assuming the same typical mass-to-light ratio of GCs in our Galaxy, i.e., $(M / L)_{V, \odot}=1.5$ for NGC 4406 and NGC 4636 or
$(M / L)_{B, \odot}=1.9$ (Illingworth 1976) in the case of NGC 4374. For NGC 4636, we also used the mass function that represents its present distribution of GCs (see Sect. 3.8). For the remaining galaxies (NGC 1400, NGC 1407, NGC 4472, NGC 3258, and NGC 3268), there is no more reliable way to estimate the total mass of "lost" GCs than by adopting as a "fiducial" reference value of their mean mass, $\left\langle m_{1}\right\rangle$, a value, $\left\langle m_{\mathrm{MW}}\right\rangle=3.3 \times 10^{5} M_{\odot}$, which is a reliable estimate of the present mean GC mass in our Galaxy, as obtainable from their LF (Abraham \& Van Den Bergh 1995).

\section{Data and results}

The data studied in this paper were collected from the literature. We analyse data of a set of 8 galaxies for which the GC content and the radial profile were reliably determined and that are suitable to our purposes.

The galaxies are: NGC 1400, NGC 1407, NGC 4472 (M 49), NGC 3268, NGC 3258, NGC 4374, NGC 4406, and NGC 4636. These galaxies add to the set of seventeen galaxies (Milky Way, M 31, M 87, NGC 1379, NGC 1399, NGC 1404, NGC 1427, NGC 1439, NGC 1700, NGC 4365, NGC 4494, NGC 4589, NGC 5322, NGC 5813, NGC 5982, NGC 7626, IC 1459) whose GCS radial profiles were compared with those of previous papers (McLaughlin 1995; Capuzzo-Dolcetta \& Vignola 1997; Capuzzo-Dolcetta \& Tesseri 1999; Capuzzo-Dolcetta \& Donnarumma 2001).

\subsection{NGC 1400}

The surface density profile of this galaxy is presented by Forbes et al. (2006) (hereafter F06), who fitted it by means of a power law $\Sigma_{\mathrm{s}}(r) \propto r^{-1.88}$. This fitting law is reliable outside the galactic core, i.e., for $r>r_{\mathrm{b}}=0.0055 \operatorname{arcmin}$ (Spolaor et al. 2008). The luminosity profile of the galaxy for $r \leq r_{\mathrm{b}}$ is almost flat, and related to the external power law. We fitted the GCS distribution with a modified core model of $\Sigma_{0}=14.1 \mathrm{arcmin}^{-2}$, $r_{\mathrm{c}}=0.7$ arcmin, and $\gamma=0.88$. Integrating $\Sigma_{\mathrm{GC}}(r)$ in the radial range where GCs are observed, i.e., from $r_{\min }=0$ arcmin to $R=2.8 \operatorname{arcmin}$, we obtain $N=73$ to be the present number of GCs in NGC 1400. The initial GCS distribution can be approximated by:

$\Sigma_{\mathrm{GC}, 0}(r)= \begin{cases}1.37 \times 10^{5} \operatorname{arcmin}^{-2} & r \leq r_{\mathrm{b}} \\ 7.76 r^{-1.88} & r>r_{\mathrm{b}}\end{cases}$

(see Fig. 1).

Using the general method described in Sect. 2 and the estimated value $r_{\max }=2.3 \mathrm{arcmin}$, we find that the number of missing clusters in this galaxy is $N_{1}=183$, i.e., about $71 \%$ of the initial population of globular clusters, $N_{\mathrm{i}}=N_{\mathrm{l}}+N=256$. An estimate of the mass lost by the GCS is $M_{1}=N_{1}\left\langle m_{\mathrm{MW}}\right\rangle=$ $6.04 \times 10^{7} M_{\odot}$.

\subsection{NGC 1407}

As for NGC 1400, data for both this galaxy and its GCS were taken from F06. The luminosity profile of the galactic stars was fitted by a power law, $\Sigma_{\mathrm{s}}(r) \propto r^{-1.42}$. This power law fit is inaccurate in the inner region, where the luminosity profile exhibits a core of radius $r_{\mathrm{b}} \simeq 0.045 \mathrm{arcmin}$ (Spolaor et al. 2008). As for NGC 1400, we thus assume, for $r \leq r_{\mathrm{b}}$, that a flat distribution matches the external power law. In this case, the GCS modified core model has $\Sigma_{0}=17.8 \operatorname{arcmin}^{-2}, r_{\mathrm{c}}=1.02 \mathrm{arcmin}$, and 

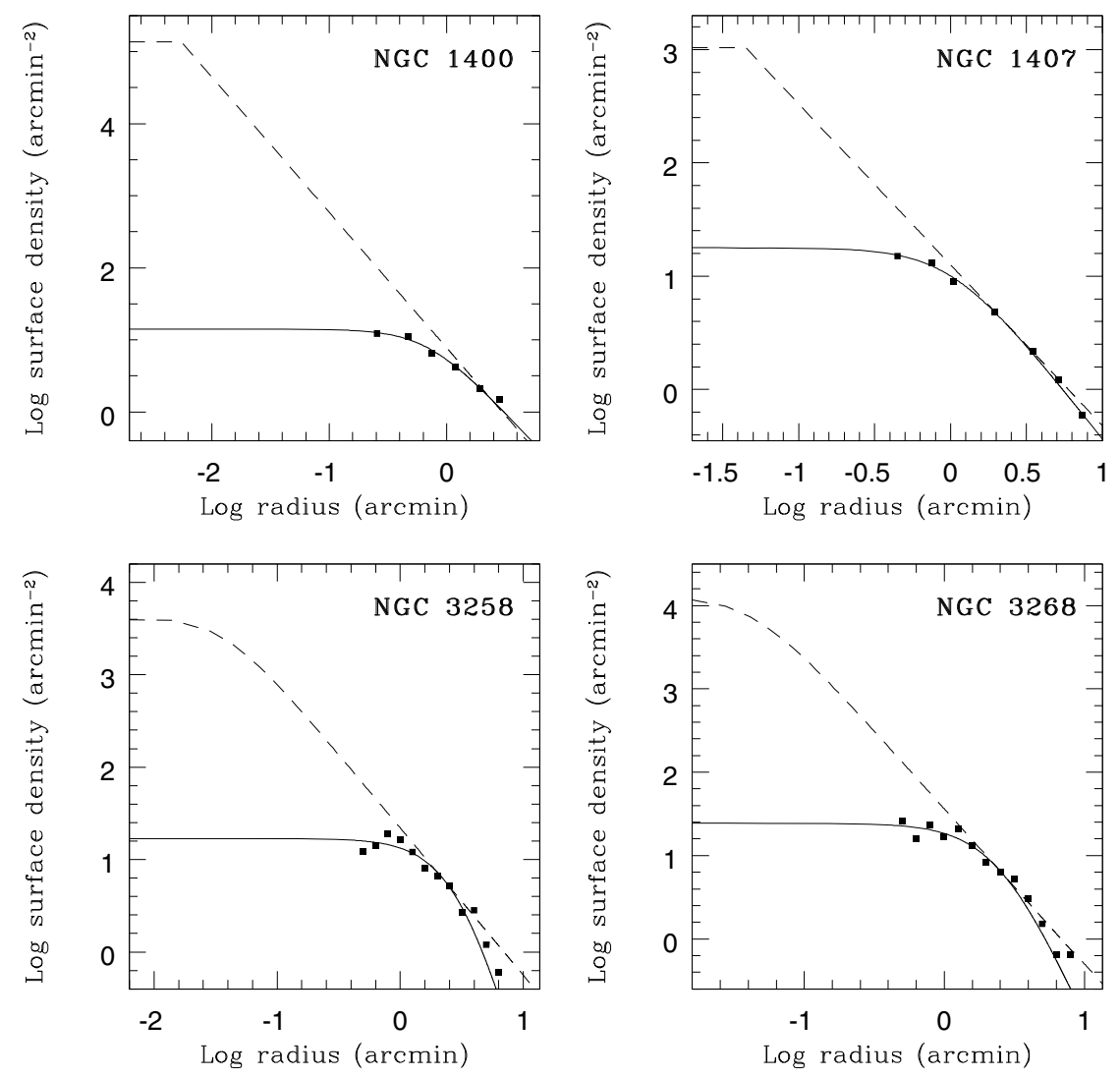

Fig. 1. Surface number density for NGC 1400, NGC 1407, NGC 3258 and NGC 3268. Black squares represent the observed GC distributions; the solid lines are their modified core model fits. The dashed curves are the surface brightness profiles of the underlying galaxy (a power law and a central flat core for NGC 1400 and NGC 1407, and a modified Hubble law for NGC 3258 and NGC 3268), vertically normalized to match the radial profile of the GCS in the outer regions.

$\gamma=0.85$ as more suitable parameters. The normalizing vertical offset of the stellar profile leads to

$\Sigma_{\mathrm{GC}, 0}(r)= \begin{cases}1.04 \times 10^{3} \operatorname{arcmin}^{-2} & r \leq r_{\mathrm{b}} \\ 12.6 r^{-1.42} & r>r_{\mathrm{b}}\end{cases}$

(see Fig. 1).

Integrating the difference between the GCS "initial" and present radial profiles in the galactic regions where these differ, i.e., up to $r_{\max }=2.34 \operatorname{arcmin}$ (see Eq. (2)), we obtain $N_{1}=84$. The present number of GCs obtained by integrating $\Sigma_{\mathrm{GC}}(r)$ in the radial range covered by the observations (i.e., from 0 to $R=7.3 \mathrm{arcmin}$ ) is $N=314$. The GCS has therefore lost $21 \%$ of its initial population, $N_{\mathrm{i}}=398$.

In this case, we can evaluate the mass lost by the system to be $M_{1}=N_{1}\left\langle m_{\mathrm{MW}}\right\rangle=2.77 \times 10^{7} M_{\odot}$.

\subsection{NGC 3258}

The GCS density profile data for this galaxy are discussed by Dirsch, Richtler \& Bassino (2003).

The closest core model fit is given by the values $\Sigma_{0}=$ $16.9 \operatorname{arcmin}^{-2}, r_{\mathrm{c}}=3.1 \mathrm{arcmin}$, and $\gamma=2.4$. The stellar luminosity profile, within 5 arcmin, following Dirsch et al. (2003), is well represented by another modified core model fitting law. By mean of the usual procedure, the initial GCS profile is found to be most accurately described by:

$\Sigma_{\mathrm{GC}, 0}(r)=\frac{4365}{\left[1+\left(\frac{r}{0.036}\right)^{2}\right]^{0.796}} \operatorname{arcmin}^{-2}$.
The present number of GCs is $N=343$ (with $R=7.94$ arcmin). Performing the surface integral of the difference between the initial and present distribution in the radial range up to $r_{\max }=$ 2.51 arcmin (see Eq. (2)), we have $N_{1}=220$, corresponding to $39 \%$ of the initial GCS population, $N_{\mathrm{i}}=563$. $10^{7} M_{\odot}$.

For this galaxy, we obtained $M_{1}=N_{1}\left\langle m_{\mathrm{MW}}\right\rangle=7.26 \times$

\subsection{NGC 3268}

As for NGC 3268, the GC distribution of NGC 3258 is taken from Dirsch et la. (2003).

The resulting core model fit parameters have the values $\Sigma_{0}=$ $24.4 \mathrm{arcmin}^{-2}, r_{\mathrm{c}}=2.6 \mathrm{arcmin}$ and $\gamma=1.9$. The analytical fit to the luminosity profile (within 5 arcmin) of the galaxy is, again, obtained with the same law (modified core model) as used for the cluster density distribution (Dirsch et al. 2003). After the vertical translation, we have

$\Sigma_{\mathrm{GC}, 0}(r)=\frac{13490}{\left[1+\left(\frac{r}{0.044}\right)^{2}\right]^{0.936}} \operatorname{arcmin}^{-2}$

Integrating $\Sigma_{\mathrm{GC}}(r)$ from $r_{\min }=0$ arcmin to $R=7.94$ arcmin, we infer $N=505$ to be the present GC number.

The number of globular clusters lost is found to be $N_{1}=612$ (from 0 to $r_{\max }=2.51$ arcmin), which corresponds to about $55 \%$ of the initial abundance, $N_{\mathrm{i}}=1117$.

As in other cases, also for this galaxy, when evaluating the mean mass of lost GCs in NCG 3268, we have to assume that $\left\langle m_{1}\right\rangle=\left\langle m_{\mathrm{MW}}\right\rangle$, obtaining $M_{1}=N_{1}\left\langle m_{\mathrm{MW}}\right\rangle=2.02 \times 10^{8} M_{\odot}$. 

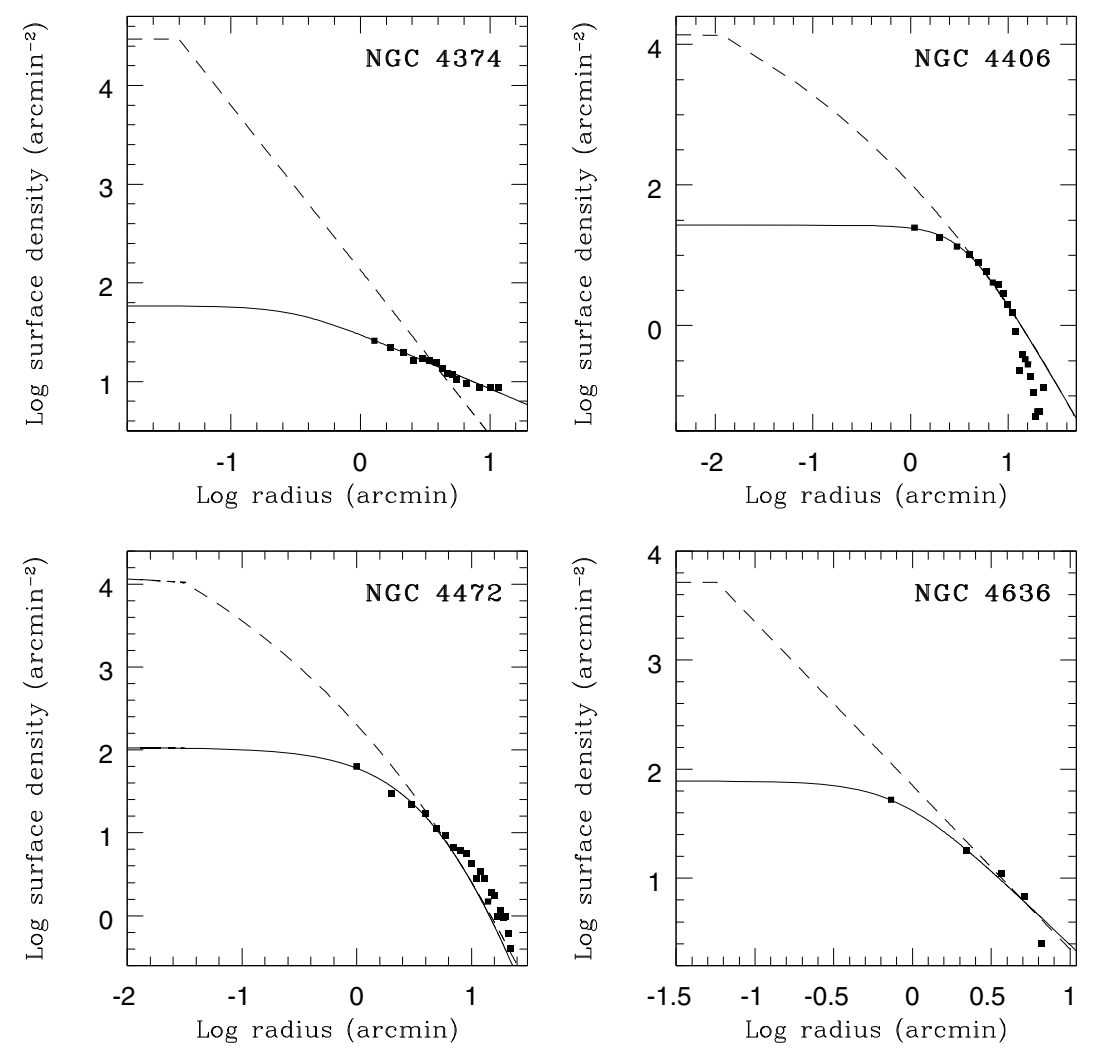

Fig. 2. Surface number density for NGC 4374, NGC 4406, NGC 4472 (M 49) and NGC 4636. Black squares represent the observed GC distributions; the solid lines are their modified core model fit (except for NGC 4472, see Sect. 3.7). The dashed curves are the surface brightness profile of the underlying galaxy (a power law and a central flat core for NGC 4374 and NGC 4636, and a Sersic core model for NGC 4406 and NGC 4472), vertically normalized to match the radial profile of the cluster system in the outer regions.

\subsection{NGC 4374 (M 84)}

Gomez \& Richtler (2004) studied the GCS of this giant elliptical galaxy, using photometry in the $B$ and $R$ bands, to determine its radial surface distribution.

In this case, contrarily to all the other galaxies discussed here, the profile of the GC number density is flatter than that of the galaxy light (see Fig. 2). This may be due to tidal interaction with the nearby NGC 4406 galaxy, which shows, indeed, a sort of depletion of the outer GC population. The best modified core model fit to the GC data is given by $\Sigma_{0}=58.4 \mathrm{arcmin}^{-2}$, $r_{\mathrm{c}}=0.31$ arcmin, and $\gamma=0.278$.

The galaxy light is characterized by a central core of radius $r_{\mathrm{b}} \simeq 0.0398$ arcmin (Lauer et al. 2007); for $r>r_{\mathrm{b}}$, it is well fitted by the power law $\Sigma_{\mathrm{s}}(r) \propto r^{-1.67}$ (Gomez \& Richtler 2004). Figure 2 shows a clear departure of the GCS from the stellar profile in the galactic outskirts which is not a surprise because it occurs beyond the galactic apparent radius $(\sim 3.1$ arcmin, as from NASA/IPAC Extragalactic Database), i.e., in a region profoundly contaminated by interaction with the tidal field of the inner, crowded, Virgo cluster region.

The usual normalization infers

$\Sigma_{\mathrm{GC}, 0}(r)= \begin{cases}2.94 \times 10^{4} \operatorname{arcmin}^{-2} & r \leq r_{\mathrm{b}} \\ 135 r^{-1.67} & r>r_{\mathrm{b}},\end{cases}$

to be the GCS initial radial profile. Integrating our core model to $R=11.8$ arcmin, we find $N=4655$ to be the present number of GCs. The usual integration of the difference between the initial and present GC distribution (Eq. (2) with $r_{\max }=3.84$ arcmin) leads to $N_{1}=2361$. Hence, NGC 4374 has lost $34 \%$ of its initial population of globular clusters, $N_{\mathrm{i}}=7016$.

In the case of NGC 4374, the value of the mean mass of a GC was evaluated using the GCLF in the $R$ band given by
Gomez \& Richtler (2004). The mean color $\left\langle(B-R)_{0}\right\rangle=1.18$ of GCs in this galaxy (Gomez \& Richtler 2004) allows us to estimate the mean $B$ absolute magnitude and the mean luminosity of GCs in the $B$ band, $\left\langle\left(L / L_{B}\right)_{\odot}\right\rangle$, by assuming that $m-M=31.61$ (Gomez \& Richtler 2004), resulting in $\left\langle\left(L / L_{B}\right)_{\odot}\right\rangle=1.75 \times 10^{5}$ (with $M_{B, \odot}=5.47$, Cox 2000).

Adopting the mass-to-light ratio $(M / L)_{B, \odot}=1.9$ obtained by Illingworth (1976) for 10 galactic globular clusters, we find that $\left\langle m_{1}\right\rangle=3.33 \times 10^{5} M_{\odot}$ and $M_{1}=N_{1}\left\langle m_{1}\right\rangle=7.86 \times 10^{8} M_{\odot}$.

\subsection{NGC 4406 (VCC 881)}

NGC 4406 is another giant elliptical in Virgo whose GCS was studied using data acquired by the Mosaic Imager on the $4 \mathrm{~m}$ Mayall telescope at the KPNO (Rhode \& Zepf 2004) in the $B$, $V$, and $R$ bands. The resulting best-fit core model is characterized by $\Sigma_{0}=26.76 \mathrm{arcmin}^{-2}, r_{\mathrm{c}}=3.52 \mathrm{arcmin}$, and $\gamma=1.19$. The core model fit does not reproduce the outer $(r>50 \mathrm{kpc}) \mathrm{GCS}$ distribution satisfactorily (see Fig. 2). Since NGC 4406 has a massive close companion (NGC 4374), it is possible that its GCS shows signs of tidal truncation due either to interaction with its companion or the general tidal field of the Virgo cluster (see Rhode \& Zepf 2004).

The galaxy star luminosity distribution, according to Ferrarese et al. (2006), is well reproduced by a Sersic core model (Trujillo et al. 2004), i.e., by

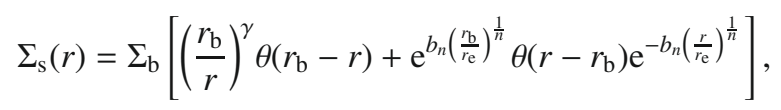

where $\Sigma_{\mathrm{s}}\left(r_{\mathrm{b}}\right)=\Sigma_{\mathrm{b}}, \theta(x)$ is the usual Heaviside function, $r_{\mathrm{b}}$ (break radius) divides the profile into an inner $\left(r \leq r_{\mathrm{b}}\right)$ power-law region and an outer $\left(r \geq r_{\mathrm{b}}\right)$ exponential region; $r_{\mathrm{e}}$ is the "effective" 
radius and $b_{n}=1.992 n-0.3271$, where $n$ is a free fitting parameter.

For NGC 4406, the parameter values are (Ferrarese et al. 2006): $\gamma=0.021, n=7.016, b_{n}=13.649$, and $r_{\mathrm{e}}=411.84 \operatorname{arcsec}(=6.864 \operatorname{arcmin}), r_{\mathrm{b}}=0.72 \operatorname{arcsec}$ $(=0.0127$ arcmin $)$. Following a vertical translation, the initial GCS radial profile is given by

$$
\begin{aligned}
\Sigma_{\mathrm{GC}, 0}(r)= & 13490\left[\left(\frac{0.012}{r}\right)^{0.021} \theta(0.012-r)\right. \\
& \left.+250 \theta(r-0.012) \mathrm{e}^{-13.649\left(\frac{r}{6.864}\right)^{0.142}}\right] .
\end{aligned}
$$

In this case we note that, unique to the sample of 8 galaxies examined in this paper, the stellar profile in the external zone decreases more slowly than that of the GCS, probably because of GC stripping from both the companion NGC 4374 and the general tidal field in the dense central Virgo cluster region where NGC 4406 is located.

By integrating the present distribution of GCs from 0 arcmin to $R=24$ arcmin, we find that $N=2850$. The surface integral given in Eq. (2), with $r_{\max }=5.36$ arcmin, infers the number of globular clusters lost, $N_{1}=1359$, i.e., about $32 \%$ of the initial GC population $\left(N_{\mathrm{i}}=4209\right)$.

Using the GCLF of this galaxy and its distance modulus $m-$ $M=31.12$ (Rhode \& Zepf 2004), we evaluated the mean value of the absolute GC V magnitude, $\left\langle M_{V}\right\rangle=-8.42$ corresponding to the mean luminosity $\left\langle L / L_{\odot}\right\rangle_{V}=1.98 \times 10^{5}\left(M_{V, \odot}=4.82\right.$ from Cox 2000).

Assuming $(M / L)_{V, \odot}=1.5$, we obtain $\left\langle m_{1}\right\rangle=2.97 \times 10^{5} M_{\odot}$. This estimate infers the value of the mass lost by the GCS to be $M_{1}=N_{1}\left\langle m_{1}\right\rangle=4.04 \times 10^{8} M_{\odot}$.

\subsection{NGC 4472 (M 49)}

Data of the GC distribution in this giant elliptical galaxy in Virgo were taken from Rhode \& Zepf (2001). The galaxy light is well fitted by a Sersic core model (Eq. (8)), whose parameters, determined by Ferrarese et al. (2006), are: $\gamma=0.086$, $n=5.503, b_{n}=10.635, r_{\mathrm{e}}=208.01 \operatorname{arcsec}(=3.47 \operatorname{arcmin})$, and $r_{\mathrm{b}}=1.94 \operatorname{arcsec}(=3.47 \operatorname{arcmin})$.

In this case, the core model profile does not provide a good approximation to the observed GCS distribution. A closer fit is given by one of the family of galaxy models (gamma-models) developed by Dehnen (1993): $\Sigma_{\mathrm{GC}}=\Sigma_{0}\left(r / r_{\mathrm{c}}\right)^{-\gamma}\left(1+r / r_{\mathrm{c}}\right)^{\gamma-4}$. Here we assume that $\gamma=0$, as suggested by Côté et al. (2003). Using this law, we found that

$\Sigma_{\mathrm{GC}}(r)=107.0\left(1+\frac{r}{8.465}\right)^{-4}$.

After the usual vertical translation, we obtained

$$
\begin{aligned}
\Sigma_{\mathrm{GC}, 0}(r)= & 10471\left[\left(\frac{0.0323}{r}\right)^{0.086} \theta(0.0323-r)\right. \\
& \left.+94.7 \theta(r-0.0323) \mathrm{e}^{-10.635\left(\frac{r}{3.47}\right)^{0.18}}\right] .
\end{aligned}
$$

Performed with $r_{\max }=7$ arcmin, the surface integral (Eq. (2)), infers that $N_{1}=4792$. Integrating $\Sigma_{\mathrm{GC}}(r)$ to $R=22$ arcmin, we have that $N=6514$. Hence, M 49 has lost $44 \%$ of the initial population of GCs, i.e., $N_{\mathrm{i}}=14752$.

As for NGC 1400 and NGC 1407, a more robust estimate of $\left\langle m_{1}\right\rangle$ is not available, so we evaluate $M_{1}$ to be $M_{1}=N_{1}\left\langle m_{\mathrm{MW}}\right\rangle=$ $1.58 \times 10^{9} M_{\odot}$.
Table 1. GCS radial distribution parameters.

\begin{tabular}{lccccc}
\hline \hline Galaxy & $\Sigma_{0}$ & $r_{\mathrm{c}}$ & $\gamma$ & $r_{\max }$ & $R$ \\
\hline NGC 1400 & 14.1 & 0.7 & 0.88 & 2.3 & 2.8 \\
NGC 1407 & 17.8 & 1.02 & 0.85 & 2.34 & 7.3 \\
NGC 3258 & 16.9 & 3.1 & 2.4 & 2.51 & 7.94 \\
NGC 3268 & 24.4 & 2.6 & 1.9 & 2.51 & 7.94 \\
NGC 4374 & 58.4 & 0.31 & 0.278 & 3.84 & 11.8 \\
NGC 4406 & 26.76 & 3.52 & 1.19 & 5.36 & 24 \\
NGC 4472 & 107 & 8.47 & 0 & 7 & 22 \\
NGC 4636 & 77.66 & 0.823 & 0.691 & 4.75 & 6.6 \\
\hline
\end{tabular}

Column 1: galaxy name; Cols. 2, 3 and 4: parameters of the modified core model fit for all the galaxies studied; Col. 5: superior limit of the integral giving the number of lost GCs $\left(r_{\max }\right)$; Col. 6: superior limit of the integral performed to estimate the present number of GCs $(R)$, where $\Sigma_{0}$ is in $\operatorname{arcmin}^{-2}$ and $r_{\mathrm{c}}, r_{\max }$ and $R$ are in arcmin.

\subsection{NGC 4636}

The GC content of this galaxy was studied by Kissler Patig et al. (1994). The modified core model fit has $\Sigma_{0}=77.66 \mathrm{arcmin}^{-2}$, $r_{\mathrm{c}}=0.823$ arcmin, and $\gamma=0.691$ as optimal parameter values.

The galactic light profile shows an inner flat distribution (a core with radius $r_{\mathrm{b}} \simeq 0.0573$ arcmin Lauer et al. 2007), while for $r>r_{\mathrm{b}}$ the light distribution is well fitted by the power-law fit $\Sigma_{\mathrm{s}}(r) \propto r^{-1.5}$ (Kissler Patig et al. 1994).

The vertical translation of the stellar profile gives the initial GCS profile:

$\Sigma_{\mathrm{GC}, 0}(r)= \begin{cases}5.16 \times 10^{3} \operatorname{arcmin}^{-2} & r \leq r_{\mathrm{b}} \\ 70.8 r^{-1.5} & r>r_{\mathrm{b}}\end{cases}$

Integrating the present surface density profile of the GCS to $R=6.6 \operatorname{arcmin}$, we obtain $N=1411$. Performing the surface integral given in Eq. (2) (with $r_{\max }=4.75$ arcmin), we estimate that the number of GCs that have disappeared is $N_{1}=746$, i.e., $35 \%$ of the initial population, $N_{\mathrm{i}}=2157$. For this galaxy, we obtained two different estimates of the mass lost by the GCS, starting from data taken from Kissler Patig et al. (1994). The first estimate was obtained using the GCLF (Kissler Patig et al. 1994). As for NGC 4406, we calculated the mean absolute V magnitude of GCs, $\left\langle M_{V}\right\rangle=-8.07$, (given that $m-M=31.2$ according to Kissler Patig et al. 1994). Assuming for GCs in NGC 4636 the same $M / L_{V}$ ratio of galactic GCs, $\left(M / L_{V}\right)_{\odot}=1.5$, the deduced mean luminosity of NGC $4636 \mathrm{GCs},\left\langle L / L_{\odot}\right\rangle_{V}=1.43 \times 10^{5}$, infers $\left\langle m_{1,1}\right\rangle=2.15 \times 10^{5} M_{\odot}$, and $M_{1,1}=N_{1}\left\langle m_{1,1}\right\rangle=1.29 \times$ $10^{8} M_{\odot}$. Another estimate is found using the mass distribution of GCs obtained in Kissler Patig et al. (1994) by transforming the magnitude bins of the GCLF candidates into masses using the relation given by Mandushev et al. (1991), $\log \left(M / M_{\odot}\right)=$ $-0.46 M_{V}+1.6$, (corresponding to a mean mass-to-light ratio $\left.(M / L)_{V, \odot} \simeq 2.0\right)$. Knowing the mass distribution, we can directly calculate the mean mass of GCs, $\left\langle m_{1,2}\right\rangle=3.79 \times 10^{5} M_{\odot}$, and thus $M_{1,2}=N_{1}\left\langle m_{1,2}\right\rangle=2.97 \times 10^{8} M_{\odot}$. $10^{8} M_{\odot}$.

The average of our $M_{1,1}$ and $M_{1,2}$ values gives $M_{1}=2.22 \times$

To provide some insight into the effects of using different photometry for the galaxy stellar profile, we adopted for the whole radial extension of NGC 4636 the "Nuker" stellar profile given in Lauer et al. (2007) and re-evaluated the $N_{1}$ value. In this way, we obtained $N_{1}=748$ (compared the previous value of 645) with $r_{\max }=2.2$ arcmin (previously $4.75 \operatorname{arcmin}$ ), where a $14.8 \%$ relative variation.

Tables 1 and 2 summarize the parameters of the radial-profile fitting functions for the studied galaxies, while Table 3 presents 
Table 2. Galactic luminosity fitting parameters.

\begin{tabular}{lccccccccc}
\hline \hline Galaxy & Model & $\eta$ & $r_{\mathrm{b}}$ & $r_{\mathrm{e}}$ & $b_{n}$ & $n$ & $\alpha$ & $\beta$ & $\gamma$ \\
\hline NGC 1400 & lc & 7.76 & 0.0055 & - & - & - & 1.88 & - & - \\
NGC 1407 & lc & 12.6 & 0.045 & - & - & - & 1.42 & - & - \\
NGC 3258 & cM & 4365 & 0.036 & - & - & - & - & - & 0.796 \\
NGC 3268 & cM & 13490 & 0.044 & - & - & - & - & - & 0.936 \\
NGC 4374 & lc & 135 & 0.0398 & - & - & - & 1.67 & - & - \\
NGC 4406 & cS & 13490 & 0.012 & 6.86 & 13.649 & 7.02 & - & - & 0.021 \\
NGC 4472 & cS & 10471 & 0.0323 & 3.47 & 10.635 & 5.503 & - & - & 0.086 \\
NGC 4636 & lc & 70.8 & 0.0573 & - & - & - & 1.5 & - & - \\
\hline
\end{tabular}

Column 1: galaxy name; Col. 2 label identifying the galaxy light profile model (lc = linear with a flat core in the inner region, $\mathrm{cS}=\mathrm{core}-\mathrm{Sérsic}$, $\mathrm{cM}=$ modified core model); Cols. 3-10: parameters of the various profile models (see Sects. 2 and 3 for details), where $\eta$ is in arcmin ${ }^{-2}$ and $r_{\mathrm{b}}$ and $r_{\mathrm{e}}$ are in arcmin.

Table 3. Deduced parameters for the various GCSs.

\begin{tabular}{lrrrrrrr}
\hline \hline Galaxy & $N$ & $N_{\mathrm{i}}$ & $N_{\mathrm{l}}$ & $\delta N$ & $\epsilon_{1}$ & $M_{\mathrm{i}}$ & $M_{1}$ \\
\hline NGC 1400 & 73 & 256 & 183 & 0.71 & 0.40 & $8.45 \times 10^{7}$ & $6.04 \times 10^{7}$ \\
NGC 1407 & 314 & 398 & 84 & 0.21 & 0.12 & $1.31 \times 10^{8}$ & $2.77 \times 10^{7}$ \\
NGC 3258 & 343 & 563 & 220 & 0.39 & 0.16 & $2.47 \times 10^{8}$ & $7.26 \times 10^{7}$ \\
NGC 3268 & 505 & 1117 & 612 & 0.55 & 0.12 & $3.69 \times 10^{8}$ & $2.02 \times 10^{8}$ \\
NGC 4374 & 4655 & 7016 & 2361 & 0.34 & 0.050 & $2.34 \times 10^{9}$ & $7.86 \times 10^{8}$ \\
NGC 4406 & 2850 & 4209 & 1359 & 0.32 & 0.23 & $1.25 \times 10^{9}$ & $4.04 \times 10^{8}$ \\
NGC 4472 & 6514 & 14752 & 4792 & 0.44 & 0.14 & $4.87 \times 10^{9}$ & $1.58 \times 10^{9}$ \\
NGC 4636 & 1411 & 2157 & 746 & 0.35 & 0.11 & $6.41 \times 10^{8}$ & $2.22 \times 10^{8}$ \\
\hline
\end{tabular}

Column 1: galaxy name; Cols. 2-8: the present number of GCs $(N)$, its initial value $\left(N_{\mathrm{i}}\right)$, the number of GCs lost $\left(N_{\mathrm{l}}\right)$, the percentage of GCs lost and the estimated relative error on $N_{1}\left(\epsilon_{1}\right.$, see Appendix A), the estimate of the initial mass of both the entire GCS $\left(M_{\mathrm{i}}\right)$ and the mass lost by each $\operatorname{GCS}\left(M_{1}\right) . M_{\mathrm{i}}$ and $M_{1}$ are in solar masses.

the results in terms of the estimated number and mass of GCs lost.

For the error estimates of Table 3 (see Appendix A), we note that a $1 \%$ error in $r_{\max }$ contributes between $1.4 \%$ (NGC 1400) and $26 \%$ (NGC 1407) to the total error. For the greatest difference in the slope of the external GCS and stellar profiles (NGC 4374), the contribution of a $1 \%$ indeterminacy in $r_{\text {max }}$ corresponds to $22 \%$ of the total error.

\section{The correlation between $M_{l}, M_{v}$, and $M_{b h}$}

The evolutionary explanation of the difference between the initial and present GC distribution implies a correlation between the (supposed) mass lost by GCS, with the mass of the galactic central supermassive black hole $\left(M_{\mathrm{bh}}\right)$ and, probably, with the host galaxy luminosity $\left(M_{V}\right)$. Table 4 reports the whole set of galaxies for which we have the estimate of $M_{V}, M_{\mathrm{bh}}$, and $M_{1}$.

Figure 3 shows a plot of the data given in Table 4 . For some galaxies, two estimates of $M_{V}$ and/or $M_{\mathrm{bh}}$ were available. In these cases, we took from Table 4 the entries in the first of the two rows to plot in Fig. 3. The figure clearly indicates an increasing trend of $M_{1}$ as function of $M_{\mathrm{bh}}$ (left panel) and of $M_{V}$ (right panel).

In particular, the linear fit of data in the left panel is given by $\log M_{1}=a \log M_{\mathrm{bh}}+b$ with $a \pm \sigma(a)=0.56 \pm 0.14$ and $b \pm$ $\sigma(b)=3.3 \pm 1.2$, giving $\chi^{2}=3.8$. The alternative, exponential fit gives $\log M_{1}=\alpha \exp \left(\log M_{\mathrm{bh}}\right)+\beta$, where $\alpha \pm \sigma(\alpha)=(7.6 \pm$ $2.7) \times 10^{-5}$ and $\beta \pm \sigma(\beta)=7.61 \pm 0.22$ and $\chi^{2}=4.6$.

For the 9 galaxies for which alternative data are available we re-evaluated the linear and exponential fit to data using the second row values in Table 4, obtaining $a \pm \sigma(a)=0.51 \pm 0.16$ and $b \pm \sigma(b)=3.7 \pm 1.3$, where $\chi^{2}=4.1$ for the linear fit, and then for the exponential fit, $\alpha \pm \sigma(\alpha)=(1.04 \pm 0.30) \times 10^{-4}$ and $\beta \pm \sigma(\beta)=7.44 \pm 0.22$, where $\chi^{2}=4.0$. The differences between the fit coefficients are insignificant.

The least squares, straight-line, fit to the whole set of data shown in the right panel of Fig. 3 is given by $\log M_{1}=a M_{V}+b$, where $a \pm \sigma(a)=-0.55 \pm 0.10$ and $b \pm \sigma(b)=-3.8 \pm 2.2$ providing $\chi^{2}=2.6$. The exponential fit to the same data gives $\log M_{1}=\alpha \exp \left(-M_{V}\right)+\beta$, where $\alpha \pm \sigma(\alpha)=(1.90 \pm 0.40) \times$ $10^{-10}$ and $\beta \pm \sigma(\beta)=7.54 \pm 0.16$ and $\chi^{2}=3.4$. As before, we re-evaluated the linear and exponential fits using the second set of entries for the galaxies with two evaluations in Table 4 and found that $a \pm \sigma(a)=-0.48 \pm 0.08$ and $b \pm \sigma(b)=-2.30 \pm$ 1.70, giving $\chi^{2}=2.30$, provided the tightest linear fit, and that $\alpha \pm \sigma(\alpha)=(1.01 \pm 0.19) \times 10^{-10}$ and $\beta \pm \sigma(\beta)=7.67 \pm 0.11$ and $\chi^{2}=2.6$, provided the tightest exponential fit.

In the right panel of Fig. 3 the correlation between $M_{1}$ and $M_{V}$ reflects both an expected physical dependence of evolutionary processes acting on GCSs on the total galactic mass and, more simply, the positive correlation between $M_{\mathrm{bh}}$ and $M_{V}$ for the same set of galaxies. The $M_{\mathrm{bh}}-M_{V}$ correlation for the set of galaxies in Table 4 clearly has a positive slope, as also shown by the least squares fit in Fig. 4. The least square fit is $\log M_{\mathrm{bh}}=a M_{V}+b$ with $a \pm \sigma(a)=-0.52 \pm 0.15$ and $b \pm \sigma(b)=-2.6 \pm 3.2$, giving $\chi^{2}=5.2$. When using the alternative entries in Table 4 , the fit coefficients transform into $a \pm \sigma(a)=-0.41 \pm 0.12$ and $b \pm \sigma(b)=-0.40 \pm 2.70$, giving $\chi^{2}=5.5$.

On the astrophysical side, Capuzzo-Dolcetta \& Tesseri (1999) demonstrated how a correlation between GCS radial profiles and the parent galaxy luminosity is expected for the larger tidal disturbance caused by a more massive compact central nucleus in the galaxy, which erodes the GC distribution peaked at the galactic centre by means of frictionally decayed massive clusters. 
Table 4. Integrated magnitudes and central black hole masses for a set of galaxies for which the estimate of the mass lost from their GCSs is known.

\begin{tabular}{lcccc}
\hline \hline Galaxy & $M_{V}$ & $M_{\text {bh }}$ & $M_{1}$ & Sources \\
\hline MW & -20.60 & $3.61 \times 10^{6}$ & $1.80 \times 10^{7}$ & E05, VV00, CDV97 \\
M 31 & -19.82 & $6.19 \times 10^{7}$ & $2.30 \times 10^{7}$ & M98, CDV97 \\
$"$ & $"$ & $4.50 \times 10^{7}$ & $"$ & TR02 \\
M 87 & -22.38 & $3.61 \times 10^{9}$ & $2.33 \times 10^{9}$ & M98, CDV97 \\
$"$ & -22.95 & $3.67 \times 10^{9}$ & $"$ & KB09 \\
NGC 1427 & -20.43 & $1.17 \times 10^{8}$ & $8.86 \times 10^{7}$ & VM99, CDT99 \\
NGC 4365 & -22.06 & $7.08 \times 10^{8}$ & $7.48 \times 10^{7}$ & VM99, CDT99 \\
" & -23.63 & $3.94 \times 10^{8}$ & $"$ & KB09 \\
NGC 4494 & -20.94 & $4.79 \times 10^{8}$ & $2.98 \times 10^{7}$ & VM99, CDT99 \\
NGC 4589 & -21.14 & $3.09 \times 10^{8}$ & $7.58 \times 10^{7}$ & VM99, CDT99 \\
NGC 5322 & -21.90 & $9.77 \times 10^{8}$ & $6.51 \times 10^{7}$ & VM99, CDT99 \\
NGC 5813 & -21.81 & $2.82 \times 10^{8}$ & $1.03 \times 10^{8}$ & VM99, CDT99 \\
NGC 5982 & -21.83 & $7.94 \times 10^{8}$ & $8.86 \times 10^{7}$ & VM99, CDT99 \\
NGC 7626 & -22.34 & $1.95 \times 10^{9}$ & $3.59 \times 10^{8}$ & VM99, CDT99 \\
IC 1459 & -21.68 & $2.60 \times 10^{9}$ & $1.57 \times 10^{8}$ & Fe05, VM99, CDT99 \\
" & $"$ & $2.50 \times 10^{9}$ & $"$ & TR02 \\
NGC 1399 & -21.71 & $5.22 \times 10^{9}$ & $1.44 \times 10^{8}$ & M98, CDD01 \\
" & $"$ & $5.10 \times 10^{8}$ & $"$ & GE07 \\
NGC 1400 & -20.63 & $3.71 \times 10^{8}$ & $6.04 \times 10^{7}$ & VM99, F06, CDM \\
NGC 1407 & -21.86 & $5.55 \times 10^{8}$ & $2.77 \times 10^{7}$ & Z07, F06, CDM \\
NGC 3258 & -21.40 & $2.14 \times 10^{8}$ & $7.26 \times 10^{7}$ & BC06, D03, CDM \\
NGC 3268 & -22.07 & $4.68 \times 10^{8}$ & $2.02 \times 10^{8}$ & BC06, D03, CDM \\
NGC 4374 & -22.62 & $1.41 \times 10^{9}$ & $7.86 \times 10^{8}$ & R98, GR04, CDM \\
" & -23.63 & $8.87 \times 10^{8}$ & $"$ & KB09 \\
NGC 4406 & -22.30 & $1.40 \times 10^{8}$ & $4.04 \times 10^{8}$ & CJ93, RZ04, CDM \\
" & -22.69 & $2.29 \times 10^{8}$ & $"$ & KB09 \\
NGC 4472 & -23.10 & $2.63 \times 10^{9}$ & $1.58 \times 10^{9}$ & M98, RZ04, CDM \\
" & -23.24 & $6.10 \times 10^{8}$ & $"$ & KB09 \\
NGC 4636 & -21.70 & $3.63 \times 10^{8}$ & $2.22 \times 10^{8}$ & VM99, KR94, CDM \\
" & -22.10 & $2.06 \times 10^{9}$ & $"$ & KB09 \\
\hline & & & &
\end{tabular}

Column 1: galaxy name; Cols. 2, 3, and 4: the $V$ absolute magnitudes, the galactic central black hole masses, and the mass lost by GCSs (both in solar masses), respectively; Col. 5: bibliographic reference sources for entries in Cols. 2, 3, and 4; CDM is the present paper, the other acronyms are defined in the references. For 9 galaxies, double entries for $M_{V}$ and/or $M_{\mathrm{bh}}$ are available and given in the second row. When an entry was not modified, a " symbol is present.
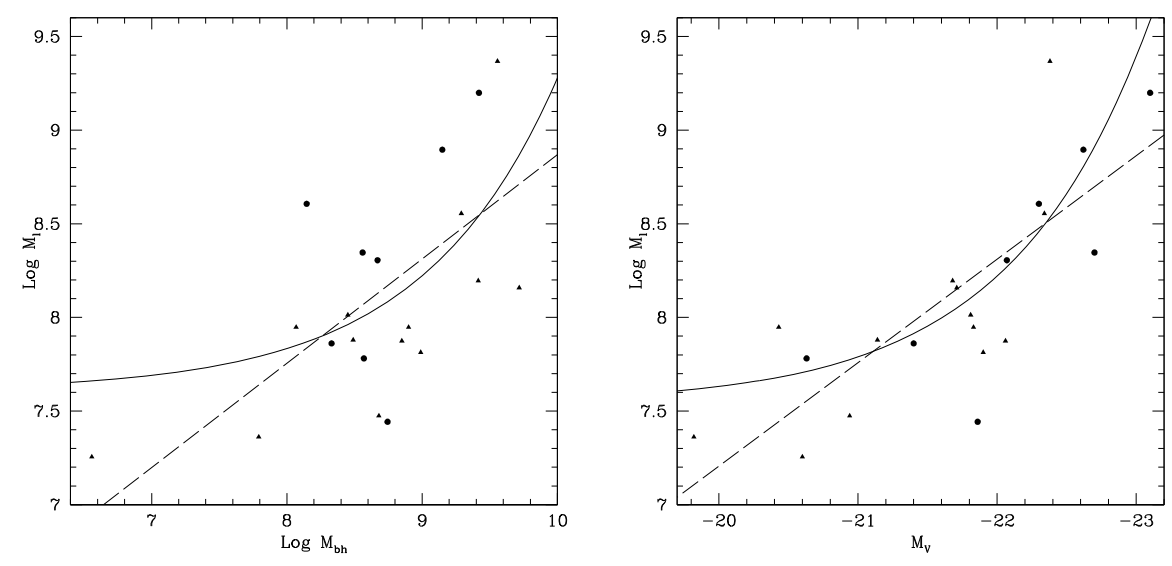

Fig. 3. The correlation between the GCS (logarithmic) mass lost and the central galactic black hole mass (left panel) and integrated $V$ magnitude of the host galaxy (right panel) for the set of galaxies in Table 4. Masses are in solar masses. Black circles represent the eight galaxies whose GCS data are discussed in this paper, black triangles refer to the others. The straight lines and curves are the approximation fits discussed in Sect. 4.

\section{Conclusions}

We have presented a comparative discussion of the radial distributions of the globular cluster systems and of stars in eight elliptical galaxies observed by various authors. We found that GCS distributions flatten towards the centre, showing a broad core in the profile, in contrast to the surrounding star field. This result agrees with many previous findings, indicating, indeed, that GCs are usually less centrally concentrated than the stars of the galaxy bulge-halo. A debate remains open on the interpretation of this observational issue. The "evolutionary" interpretation is particularly appealing; it claims that, initially, the GCS and stellar profiles were similar and, later, GCS evolved to the presently flatter distribution because of dynamical friction and tidal interactions (Capuzzo-Dolcetta 1993). In this picture, the flatter central profile is caused by the erosion of the inner GCS radial profile. Many GCs are, consequently, packed into the inner galactic region, where they influence the physics of the host 


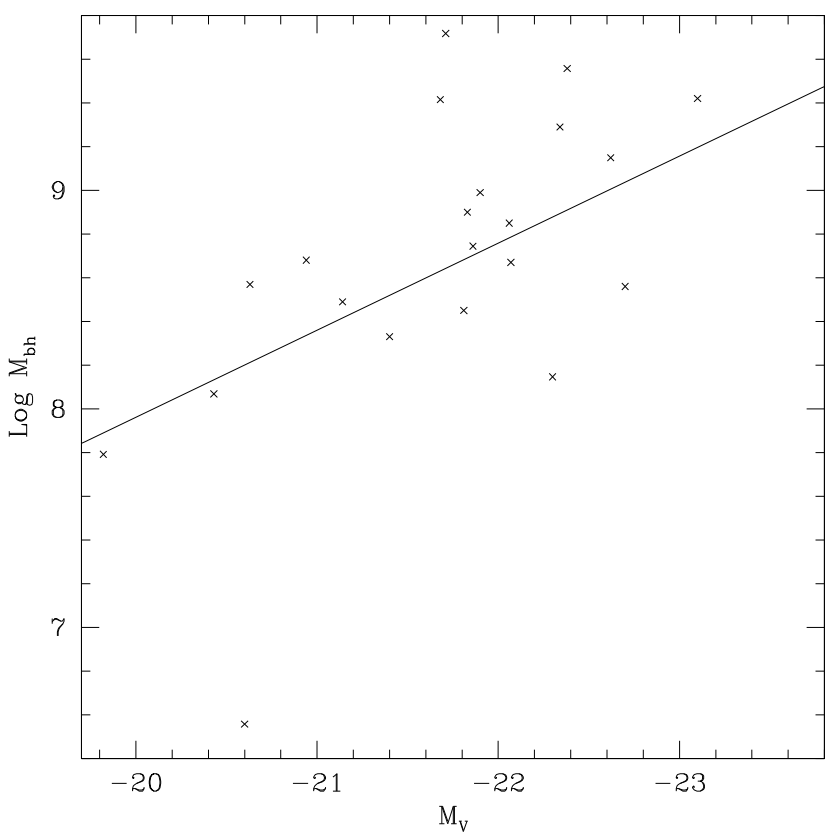

Fig. 4. The correlation between the logarithm of the central galactic black hole mass (in solar masses) and the integrated $V$ galactic magnitude (see Table 4).

galaxy. Many galaxies studied so far have massive black holes at their centres, whose mass positively correlates with our estimates of the number and mass of GCs lost. This provides a strong support of the validity of the aforementioned evolutionary scenario, together with the other clear correlations between number and mass of GC lost and their parent galaxy luminosity. In the light of these encouraging findings, we propose that much effort should be devoted towards improving the observational tests of this astrophysical scenario.

\section{Appendix A: The formal error in the estimate of the number of lost GCs}

We describe how we evaluated the errors in GCs lost, $\epsilon_{1}$, given in Table 3. As explained in Sect. 2, the number of GCs lost in the galaxies of the sample was evaluated as the integral of the difference between the (estimated) initial and present GCS radial distributions over the radial range $\left[r_{\min }, r_{\max }\right]$ where the two profiles differ. The absolute errors in $N_{1}\left(\Delta N_{1}\right)$ are given by the sum of the error in $N_{\mathrm{i}}\left(\Delta N_{\mathrm{i}}\right)$ and the error on $N(\Delta N)$, yielding the relative error $\epsilon_{1}=\frac{\Delta N_{1}}{N_{1}}$ of Table 3. We estimate $\Delta N$ and $\Delta N_{\mathrm{i}}$ as follows:

(i) To estimate of $\Delta N$, for all galaxies, except for NGC 4472, the number $N\left(r_{\min }, r_{\max }\right)$ is given by

$$
\begin{aligned}
N\left(r_{\min }, r_{\max }\right) & =2 \pi \Sigma_{0} \int_{r_{\min }}^{r_{\max }} \frac{r}{\left[1+\left(\frac{r}{r_{\mathrm{c}}}\right)^{2}\right]^{\gamma}} \mathrm{d} r \\
& =\left.\frac{\Sigma_{0} \pi\left(r^{2}+r_{\mathrm{c}}^{2}\right)}{(1-\gamma)\left[1+\left(\frac{r}{r_{\mathrm{c}}}\right)^{2}\right]^{\gamma}}\right|_{r_{\min }} ^{r_{\max }},
\end{aligned}
$$

which is a function of the parameters $\Sigma_{0}, r_{\mathrm{c}}, \gamma, r_{\min }$, and $r_{\max }$, whose indetermination is

$$
\begin{aligned}
\Delta N= & \left|\frac{\partial N}{\partial \Sigma_{0}}\right| \Delta \Sigma_{0}+\left|\frac{\partial N}{\partial r_{\mathrm{c}}}\right| \Delta r_{\mathrm{c}}+\left|\frac{\partial N}{\partial \gamma}\right| \Delta \gamma \\
& +\left|\frac{\partial N}{\partial r_{\min }}\right| \Delta r_{\text {min }}+\left|\frac{\partial N}{\partial r_{\max }}\right| \Delta r_{\max },
\end{aligned}
$$

where

$$
\begin{aligned}
\frac{\partial N}{\partial \Sigma_{0}} & =2 \pi \int_{r_{\min }}^{r_{\max }} \frac{r}{\left[1+\left(\frac{r}{r_{\mathrm{c}}}\right)^{2}\right]^{\gamma}} \mathrm{d} r=\left.\frac{\pi\left(r^{2}+r_{\mathrm{c}}^{2}\right)}{(1-\gamma)\left[1+\left(\frac{r}{r_{\mathrm{c}}}\right)^{2}\right]^{\gamma}}\right|_{r_{\min }} ^{r_{\max }}, \\
\frac{\partial N}{\partial r_{\mathrm{c}}} & =\int_{r_{\min }}^{r_{\max }} \frac{4 \pi \Sigma_{0} \gamma r^{3}}{r_{\mathrm{c}}^{3}\left[1+\left(\frac{r}{r_{\mathrm{c}}}\right)^{2}\right]^{(1+\gamma)}} \mathrm{d} r \\
& =-\left.\frac{2 \pi \Sigma_{0}\left(r_{\mathrm{c}}^{2}+\gamma r^{2}\right)}{r_{\mathrm{c}}(\gamma-1)\left[1+\left(\frac{r}{r_{\mathrm{c}}}\right)^{2}\right]^{\gamma}}\right|_{r_{\min }} ^{r_{\max }},
\end{aligned}
$$

$$
\begin{aligned}
\frac{\partial N}{\partial \gamma} & =-2 \pi \Sigma_{0} \int_{r_{\min }}^{r_{\max }} r\left[1+\left(\frac{r}{r_{\mathrm{c}}}\right)^{2}\right]^{-\gamma} \ln \left[1+\left(\frac{r}{r_{\mathrm{c}}}\right)^{2}\right] \mathrm{d} r \\
& =\left.\frac{\pi \Sigma_{0}\left(r^{2}+r_{\mathrm{c}}^{2}\right)\left\{1+(\gamma-1) \ln \left[1+\left(\frac{r}{r_{\mathrm{c}}}\right)^{2}\right]\right\}}{(\gamma-1)^{2}\left[1+\left(\frac{r}{r_{\mathrm{c}}}\right)^{2}\right]^{\gamma}}\right|_{r_{\min }} ^{r_{\max }}
\end{aligned}
$$

$\frac{\partial N}{\partial r_{\min }}=-2 \pi \Sigma_{0} \frac{r_{\min }}{\left[1+\left(\frac{r_{\min }}{r_{\mathrm{c}}}\right)^{2}\right]^{\gamma}}$,

(where we assume that $r_{\min }=0.1$ arcmin).

$\frac{\partial N}{\partial r_{\max }}=2 \pi \Sigma_{0} \frac{r_{\max }}{\left[1+\left(\frac{r_{\max }}{r_{\mathrm{c}}}\right)^{2}\right]^{\gamma}}$.

For NGC 4472 we have that:

$$
\begin{aligned}
N\left(r_{\min }, r_{\max }\right) & =2 \pi \Sigma_{0} \int_{r_{\min }}^{r_{\max }}\left(\frac{r_{\mathrm{c}}}{r}\right)^{\gamma} \frac{r}{\left[1+\frac{r}{r_{\mathrm{c}}}\right]^{4-\gamma}} \mathrm{d} r \\
& =\left.2 \pi \Sigma_{0}\left(r_{\mathrm{c}} r\right)^{2}\left(\frac{r_{\mathrm{c}}+r}{r}\right)^{\gamma} \frac{(3-\gamma) r_{\mathrm{c}}+r}{\left(6-5 \gamma+\gamma^{2}\right)\left(r_{\mathrm{c}}+r\right)^{3}}\right|_{r_{\min }} ^{r_{\max }} .
\end{aligned}
$$

This is a function of the parameters $\Sigma_{0}, r_{\mathrm{c}}, r_{\min }$, and $r_{\max }$, whose indetermination is

$$
\begin{aligned}
\Delta N= & \left|\frac{\partial N}{\partial \Sigma_{0}}\right| \Delta \Sigma_{0}+\left|\frac{\partial N}{\partial r_{\mathrm{c}}}\right| \Delta r_{\mathrm{c}}+\left|\frac{\partial N}{\partial \gamma}\right| \Delta \gamma+\left|\frac{\partial N}{\partial r_{\min }}\right| \Delta r_{\min } \\
& +\left|\frac{\partial N}{\partial r_{\max }}\right| \Delta r_{\max }
\end{aligned}
$$

where

$$
\begin{aligned}
\frac{\partial N}{\partial \Sigma_{0}} & =2 \pi \int_{r_{\min }}^{r_{\max }}\left(\frac{r_{\mathrm{c}}}{r}\right)^{\gamma} \frac{r}{\left[1+\frac{r}{r_{\mathrm{c}}}\right]^{4-\gamma} \mathrm{d} r} \\
& =\left.2 \pi\left(r_{\mathrm{c}} r\right)^{2}\left(\frac{r_{\mathrm{c}}+r}{r}\right)^{\gamma} \frac{(3-\gamma) r_{\mathrm{c}}+r}{\left(6-5 \gamma+\gamma^{2}\right)\left(r_{\mathrm{c}}+r\right)^{3}}\right|_{r_{\text {min }}} ^{r_{\max }},
\end{aligned}
$$




$$
\begin{aligned}
\frac{\partial N}{\partial r_{\mathrm{c}}}= & 2 \pi \Sigma_{0} \int_{r_{\min }}^{r_{\max }}\left[\frac{(\gamma-4) r^{2}}{r_{\mathrm{c}}^{2}}\left(\frac{r_{\mathrm{c}}}{r}\right)^{\gamma}\left(1+\frac{r}{r_{\mathrm{c}}}\right)^{\gamma-5}\right. \\
& \left.+\gamma\left(\frac{r_{\mathrm{c}}}{r}\right)^{\gamma-1}\left(1+\frac{r}{r_{\mathrm{c}}}\right)^{\gamma-4}\right] \mathrm{d} r \\
= & \frac{2 \pi \Sigma_{0} r_{\mathrm{c}} r^{2}}{\left(6-5 \gamma+\gamma^{2}\right)\left(r_{\mathrm{c}}+r\right)^{4}}\left(\frac{r_{\mathrm{c}}+r}{r}\right)^{\gamma} \\
& \times\left.\left[2 r^{2}(\gamma-1)-2 r_{\mathrm{c}} r\left(\gamma^{2}-5 \gamma+4\right)-\gamma r_{\mathrm{c}}^{2}(\gamma-3)\right]\right|_{r_{\min }} ^{r_{\max }},
\end{aligned}
$$

and

$$
\begin{aligned}
\frac{\partial N}{\partial \gamma}= & 2 \pi \Sigma_{0} \int_{r_{\min }}^{r_{\max }}\left(\frac{r_{\mathrm{c}}}{r}\right)^{\gamma} r\left(1+\frac{r}{r_{\mathrm{c}}}\right)^{\gamma-4} \ln \left(1+\frac{r_{\mathrm{c}}}{r}\right) \mathrm{d} r \\
= & \frac{2 \pi \Sigma_{0}\left(r_{\mathrm{c}} r\right)^{2}}{\left(6-5 \gamma+\gamma^{2}\right) 2\left(r_{\mathrm{c}}+r\right)^{3}}\left(\frac{r_{\mathrm{c}}+r}{r}\right)^{\gamma}\left\{r_{\mathrm{c}}\left(9-6 \gamma+\gamma^{2}\right)\right. \\
& \left.+r(5-2 \gamma)-\left(6-5 \gamma+\gamma^{2}\right)\left[(\gamma-3) r_{\mathrm{c}}-r\right] \ln \left(1+\frac{r_{\mathrm{c}}}{r}\right)\right\}\left.\right|_{r_{\min }} ^{r_{\max }},
\end{aligned}
$$

$$
\frac{\partial N}{\partial r_{\min }}=-2 \pi \Sigma_{0}\left(\frac{r_{\mathrm{c}}}{r_{\min }}\right)^{\gamma} \frac{r_{\min }}{\left[1+\frac{r_{\min }}{r_{\mathrm{c}}}\right]^{4-\gamma}}
$$

$$
\frac{\partial N}{\partial r_{\max }}=2 \pi \Sigma_{0}\left(\frac{r_{\mathrm{c}}}{r_{\max }}\right)^{\gamma} \frac{r_{\max }}{\left[1+\frac{r_{\max }}{r_{\mathrm{c}}}\right]^{4-\gamma}} .
$$

The fitting parameters used to calculate $\Delta N$ are summarized in Table 1.

(ii) To estimate $\Delta N_{\mathrm{i}}$, the fitting formulas for the initial distribution of GCs changed for the various galaxies studied.

For NGC 1400, NGC 1407, NGC 4374, and NGC 4636, we have (see Sects. 3.1, 3.2, 3.5, 3.8 and Table 2 for the meanings and the values of the parameters)

$$
\begin{aligned}
N_{\mathrm{i}}\left(r_{\min }, r_{\max }\right) & =2 \pi \eta r_{\mathrm{b}}^{-\alpha} \int_{r_{\min }}^{r_{\mathrm{b}}} r \mathrm{~d} r+2 \pi \eta \int_{r_{\mathrm{b}}}^{r_{\max }} r^{1-\alpha} \mathrm{d} r \\
& =\left.\pi \eta r_{\mathrm{b}}^{-\alpha} r^{2}\right|_{r_{\min }} ^{r_{\mathrm{b}}}+\left.2 \pi \eta \frac{r^{2-\alpha}}{2-\alpha}\right|_{r_{\mathrm{b}}} ^{r_{\max }} .
\end{aligned}
$$

In Eq. (A.15), $\eta$ represents the parameter obtained by vertical shifting the luminosity profile.

The error $\Delta N_{\mathrm{i}}$ is thus given by

$$
\begin{aligned}
\Delta N_{\mathrm{i}}= & \left|\frac{\partial N_{\mathrm{i}}}{\partial \eta}\right| \Delta \eta+\left|\frac{\partial N_{\mathrm{i}}}{\partial r_{\mathrm{b}}}\right| \Delta r_{\mathrm{b}}+\left|\frac{\partial N_{\mathrm{i}}}{\partial \alpha}\right| \Delta \alpha+\left|\frac{\partial N_{\mathrm{i}}}{\partial r_{\min }}\right| \Delta r_{\text {min }} \\
& +\left|\frac{\partial N_{\mathrm{i}}}{\partial r_{\max }}\right| \Delta r_{\max },
\end{aligned}
$$

where

$$
\begin{aligned}
\frac{\partial N_{\mathrm{i}}}{\partial \eta} & =2 \pi r_{\mathrm{b}}^{-\alpha} \int_{r_{\min }}^{r_{\mathrm{b}}} r \mathrm{~d} r+2 \pi \int_{r_{\mathrm{b}}}^{r_{\max }} r^{1-\alpha} \mathrm{d} r \\
& =\left.\pi r_{\mathrm{b}}^{-\alpha} r^{2}\right|_{r_{\min }} ^{r_{\mathrm{b}}}+\left.2 \pi \frac{r^{2-\alpha}}{2-\alpha}\right|_{r_{\mathrm{b}}} ^{r_{\max }} . \\
\frac{\partial N_{\mathrm{i}}}{\partial r_{\mathrm{b}}} & =-2 \pi \eta \alpha r_{\mathrm{b}}^{-1-\alpha} \int_{r_{\text {min }}}^{r_{\mathrm{b}}} r \mathrm{~d} r=-\left.\pi \eta r_{\mathrm{b}}^{-1-\alpha} r^{2}\right|_{r_{\text {min }}} ^{r_{\mathrm{b}}},
\end{aligned}
$$

$$
\begin{aligned}
\frac{\partial N_{\mathrm{i}}}{\partial \alpha} & =-2 \pi \eta r_{\mathrm{b}}^{-\alpha} \int_{r_{\min }}^{r_{\mathrm{b}}} r \ln \left(r_{\mathrm{b}}\right) \mathrm{d} r-2 \pi \eta \int_{r_{\mathrm{b}}}^{r_{\max }} r^{1-\alpha} \ln (r) \mathrm{d} r \\
& =-\left.2 \pi r_{\mathrm{b}}^{-\alpha} r^{2} \ln \left(r_{\mathrm{b}}\right)\right|_{r_{\text {min }}} ^{r_{\mathrm{b}}}+\left.\frac{2 \pi \eta r^{2-\alpha}\{1+[\alpha-2] \ln (r)\}}{(\gamma-2)^{2}}\right|_{r_{\mathrm{b}}} ^{r_{\max }},
\end{aligned}
$$

In this case, we also assumed that $r_{\min }=0.1$ arcmin. For all galaxies analysed, $r_{\min }>r_{\mathrm{b}}$ and $r_{\max }>r_{\mathrm{b}}$, so we have

$\frac{\partial N_{\mathrm{i}}}{\partial r_{\min }}=-2 \pi \eta r_{\min }^{1-\alpha}$,

$\frac{\partial N_{\mathrm{i}}}{\partial r_{\max }}=2 \pi \eta r_{\max }^{1-\alpha}$

For M 49 and NGC 4406 (Sects. 3.7 and 3.6), we have

$$
\begin{aligned}
N_{\mathrm{i}}\left(r_{\min }, r_{\max }\right)= & 2 \pi \eta \int_{r_{\text {min }}}^{r_{\text {max }}} r\left\{\left(\frac{r_{\mathrm{b}}}{r}\right)^{\gamma} \theta\left(r_{\mathrm{b}}-r\right)\right. \\
& \left.+\mathrm{e}^{b_{n}\left[\left(\frac{r_{\mathrm{b}}}{r_{\mathrm{e}}}\right)^{\frac{1}{n}}-\left(\frac{r}{r_{\mathrm{e}}}\right)^{\frac{1}{n}}\right.}\right]_{\left.\theta\left(r-r_{\mathrm{b}}\right)\right\} \mathrm{d} r} \\
= & \left.\frac{2 \pi \eta}{2-\gamma} r^{2}\left(\frac{r_{\mathrm{b}}}{r}\right)^{\gamma}\right|_{r_{\text {min }}} ^{r_{\mathrm{b}}}+2 \pi \eta \int_{r_{\mathrm{b}}}^{r_{\max }} r \mathrm{e}^{b_{n}\left[\left(\frac{r_{\mathrm{b}}}{r_{\mathrm{e}}}\right)^{\frac{1}{n}}-\left(\frac{r}{r_{\mathrm{e}}}\right)^{\frac{1}{n}}\right]} \mathrm{d} r
\end{aligned}
$$

where $b_{n}=1.992 n-0.3271$. The second row of the previous expression is justified by that, for both M 49 and NGC 4406, $r_{\mathrm{b}}>r_{\min }$. Thus, the error on $N_{1}$

$$
\begin{aligned}
\Delta N_{\mathrm{i}}= & \left|\frac{\partial N_{\mathrm{i}}}{\partial \eta}\right| \Delta \eta+\left|\frac{\partial N_{\mathrm{i}}}{\partial b_{n}}\right| \Delta b_{n}+\left|\frac{\partial N_{\mathrm{i}}}{\partial r_{\mathrm{b}}}\right| \Delta r_{\mathrm{b}}+\left|\frac{\partial N_{\mathrm{i}}}{\partial \gamma}\right| \Delta \gamma \\
& +\left|\frac{\partial N_{\mathrm{i}}}{\partial r_{\mathrm{e}}}\right| \Delta r_{\mathrm{e}}+\left|\frac{\partial N_{\mathrm{i}}}{\partial n}\right| \Delta n+\left|\frac{\partial N_{\mathrm{i}}}{\partial r_{\text {min }}}\right| \Delta r_{\text {min }}+\left|\frac{\partial N_{\mathrm{i}}}{\partial r_{\text {max }}}\right| \Delta r_{\text {max }},
\end{aligned}
$$

where $\Delta b_{n}=\left|\frac{\partial b_{n}}{\partial n}\right| \Delta n$, is evaluated by the following expressions of the individual error contribution:

$$
\begin{aligned}
\frac{\partial N_{\mathrm{i}}}{\partial \eta} & =2 \pi \int_{r_{\min }}^{r_{\mathrm{b}}} r\left(\frac{r_{\mathrm{b}}}{r}\right)^{\gamma} \mathrm{d} r+2 \pi \int_{r_{\mathrm{b}}}^{r_{\max }} r \mathrm{e}^{b_{n}\left[\left(\frac{r_{\mathrm{b}}}{r_{\mathrm{e}}}\right)^{\frac{1}{n}}-\left(\frac{r}{r_{\mathrm{e}}}\right)^{\frac{1}{n}}\right]} \mathrm{d} r \\
& =\left.\frac{2 \pi}{2-\gamma} r^{2}\left(\frac{r_{\mathrm{b}}}{r}\right)^{\gamma}\right|_{r_{\min }} ^{r_{\mathrm{b}}}+2 \pi \int_{r_{\mathrm{b}}}^{r_{\max }} r \mathrm{e}^{b_{n}\left[\left(\frac{r_{\mathrm{b}}}{r_{\mathrm{e}}}\right)^{\frac{1}{n}}-\left(\frac{r}{r_{\mathrm{e}}}\right)^{\frac{1}{n}}\right]} \mathrm{d} r, \\
\frac{\partial N_{\mathrm{i}}}{\partial b_{n}} & =2 \pi \eta \int_{r_{\mathrm{b}}}^{r_{\max }} r \mathrm{e}^{b_{n}\left[\left(\frac{r_{\mathrm{b}}}{r_{\mathrm{e}}}\right)^{\frac{1}{n}}-\left(\frac{r}{r_{\mathrm{e}}}\right)^{\frac{1}{n}}\right]}\left[\left(\frac{r_{\mathrm{b}}}{r_{\mathrm{e}}}\right)^{\frac{1}{n}}-\left(\frac{r}{r_{\mathrm{e}}}\right)^{\frac{1}{n}}\right] \mathrm{d} r,
\end{aligned}
$$

$$
\begin{aligned}
\frac{\partial N_{\mathrm{i}}}{\partial r_{\mathrm{b}}}= & 2 \pi \eta \gamma \int_{r_{\min }}^{r_{\mathrm{b}}}\left(\frac{r_{\mathrm{b}}}{r}\right)^{\gamma-1} \mathrm{~d} r \\
& +2 \pi \frac{\eta b_{n}}{n r_{\mathrm{e}}} \int_{r_{\mathrm{b}}}^{r_{\max }} r \mathrm{e}^{b_{n}\left[\left(\frac{r_{\mathrm{b}}}{r_{\mathrm{e}}}\right)^{\frac{1}{n}}-\left(\frac{r}{r_{\mathrm{e}}}\right)^{\frac{1}{n}}\right]}\left(\frac{r_{\mathrm{b}}}{r_{\mathrm{e}}}\right)^{\frac{1}{n}-1} \mathrm{~d} r \\
= & \left.\frac{2 \pi \eta \gamma}{2-\gamma} r\left(\frac{r_{\mathrm{b}}}{r}\right)^{\gamma-1}\right|_{r_{\min }} ^{r_{\mathrm{b}}} \\
& +2 \pi \frac{\eta b_{n}}{n r_{\mathrm{e}}} \int_{r_{\mathrm{b}}}^{r_{\max }} r \mathrm{e}^{b_{n}\left[\left(\frac{r_{\mathrm{b}}}{r_{\mathrm{e}}}\right)^{\frac{1}{n}}-\left(\frac{r}{r_{\mathrm{e}}}\right)^{\frac{1}{n}}\right]}\left(\frac{r_{\mathrm{b}}}{r_{\mathrm{e}}}\right)^{\frac{1}{n}-1} \mathrm{~d} r,
\end{aligned}
$$




$$
\begin{aligned}
\frac{\partial N_{\mathrm{i}}}{\partial \gamma} & =2 \pi \eta \int_{r_{\min }}^{r_{\mathrm{b}}} r\left(\frac{r_{\mathrm{b}}}{r}\right)^{\gamma} \ln \left(\frac{r_{\mathrm{b}}}{r}\right) \mathrm{d} r \\
& =\left.\frac{2 \pi \eta r^{2}}{(\gamma-2)^{2}}\left(\frac{r_{\mathrm{b}}}{r}\right)^{\gamma}\left[1+(2-\gamma) \ln \left(\frac{r_{\mathrm{b}}}{r}\right)\right]\right|_{r_{\min }} ^{r_{\mathrm{b}}}
\end{aligned}
$$

$$
\begin{aligned}
\frac{\partial N_{\mathrm{i}}}{\partial r_{\mathrm{e}}}= & -2 \pi \frac{\eta b_{n}}{n r_{\mathrm{e}}^{2}} \int_{r_{\mathrm{b}}}^{r_{\max }} r \mathrm{e}^{b_{n}\left[\left(\frac{r_{\mathrm{b}}}{r_{\mathrm{e}}}\right)^{\frac{1}{n}}-\left(\frac{r}{r_{\mathrm{e}}}\right)^{\frac{1}{n}}\right]} \\
& \times\left[r_{\mathrm{b}}\left(\frac{r_{\mathrm{b}}}{r_{\mathrm{e}}}\right)^{\frac{1}{n}-1}-r\left(\frac{r}{r_{\mathrm{e}}}\right)^{\frac{1}{n}-1}\right] \mathrm{d} r
\end{aligned}
$$

$$
\begin{aligned}
\frac{\partial N_{\mathrm{i}}}{\partial n}= & -2 \pi \frac{\eta b_{n}}{n^{2}} \int_{r_{\mathrm{b}}}^{r_{\max }} r \mathrm{e}^{b_{n}\left[\left(\frac{r_{\mathrm{b}}}{r_{\mathrm{e}}}\right)^{\frac{1}{n}}-\left(\frac{r}{r_{\mathrm{e}}}\right)^{\frac{1}{n}}\right]} \\
& \times\left[\left(\frac{r_{\mathrm{b}}}{r_{\mathrm{e}}}\right)^{\frac{1}{n}} \ln \left(\frac{r_{\mathrm{b}}}{r_{\mathrm{e}}}\right)-\left(\frac{r}{r_{\mathrm{e}}}\right)^{\frac{1}{n}} \ln \left(\frac{r}{r_{\mathrm{e}}}\right)\right] \mathrm{d} r .
\end{aligned}
$$

Remembering that, for both NGC 4472 and NGC 4406, $r_{\min }>r_{\mathrm{b}}$ and $r_{\max }>r_{\mathrm{b}}$, we can estimate the following contributions

$$
\begin{aligned}
& \frac{\partial N_{\mathrm{i}}}{\partial r_{\min }}=-2 \pi \eta r_{\min } \exp \left\{b_{n}\left[\left(\frac{r_{\mathrm{b}}}{r_{\mathrm{e}}}\right)^{\frac{1}{n}}-\left(\frac{r_{\min }}{r_{\mathrm{e}}}\right)^{\frac{1}{n}}\right]\right\}, \\
& \frac{\partial N_{\mathrm{i}}}{\partial r_{\max }}=2 \pi \eta r_{\max } \exp \left\{b_{n}\left[\left(\frac{r_{\mathrm{b}}}{r_{\mathrm{e}}}\right)^{\frac{1}{n}}-\left(\frac{r_{\max }}{r_{\mathrm{e}}}\right)^{\frac{1}{n}}\right]\right\} .
\end{aligned}
$$

See Table 2 for the values of the parameters used in Eqs. (A.22)(A.31).

Finally, for NGC 3268 and NGC 3258 (see Sect. 3.4, Sect. 3.3 and Table 2 for values of the parameters), we have the same equations obtained for the present profile of all the galaxies, except NGC 4472 (see Eq. (A.1)-(A.7)). All the integrals from Eq. (A.22) to Eq. (A.29) must be calculated numerically using the values of the parameters given in Table 2 .

The results listed in Table 3 are obtained by assuming an error of $1 \%$ in each independent parameter used. Only in the case of NGC 4472, where we assumed $\gamma=0$ (Côté et al. 2003), we could not consider a value for $\Delta \gamma$ obtained by a fixed percentual variation and, thus, we adopted $\Delta \gamma=0.01$.

\section{References}

Abraham, R. G., \& Van Den Bergh, S. 1995, ApJ, 438, 218

Ashman K. M., \& Zepf, S. E. 1998, Globular Cluster Systems, Cambridge astrophysics series, 30 (Cambridge University Press, New York) Balmaverde B., \& Capetti, A. 2006, A\&A, 447, 97, BC06

Bassino, L. P., Faifer, F. R., Forte, J. C., et al. 2006, A\&A, 451, 789

Bekki K., \& Forbes, D. A. 2006, A\&A, 445, 485

Bertin G., Ciotti, L., \& Del Principe, M. 2002, A\&A, 386, 149

Capetti A., \& Balmaverde, B. 2005, A\&A, 440, 73

Capuzzo Dolcetta, R. 1993 ApJ, 415, 616
Capuzzo Dolcetta, R. 2002, ASP Conf. Ser., vol. 285, 389

Capuzzo Dolcetta, R., \& Donnarumma, I. 2001, MNRAS, 328, 645 Capuzzo Dolcetta, R., \& Tesseri, A. 1997, MNRAS, 292, 808, CDT99

Capuzzo Dolcetta, R., \& Tesseri, A. 1999, MNRAS, 308, 961

Capuzzo Dolcetta, R., \& Vicari, A. 2005, MNRAS, 356, 899

Capuzzo Dolcetta, R., \& Vignola, L. 1997, A\&A, 327, 130, CD97

Carter D., \& Jenkins, C. R. 1993, MNRAS, 263, 1049, CJ93

Côté P., McLaughlin, D. E., Cohen, J. G., \& Blakeslee, J. P., ApJ 2003, 591, 850

Cox A. N. 2000, Allen's Astrophysical Quantities, 4th edn. (Springer-Verlag)

Dehnen, W. 1993, MNRAS, 265, 250

Dirsch B., Richtler T., \& Bassino L. P. 2003, A\&A, 408, 929, D03

Eisenhauer F., Genzel, R., Alexander, T., et al. 2005, ApJ, 628, 246, E05

Elmegreen B. 1999, Ap\&SS, 269-270, 469

Fall S. M., \& Rees, M. J. 1977, MNRAS, 181, 37

Ferrarese L., \& Ford H. 2005, SSRv, 116, 523, Fe05

Ferrarese L., Côté, P., Jordán, A., et al. 2006, ApJ, 164, 334

Forbes D. A., Franx, M., Illingworth, G. D., \& Carollo, C. M. 1996, ApJ, 467, 126

Forbes D. A., Sanchez- Blazquez, P., Phan, A. T. T., et al. 2006, MNRAS, 366, 1230, F06

Forte J. C., Martinez, R. E., \& Muzzio, J. C. 1982, AJ, 87, 1465

Gómez M., \& Richtler, T. 2004, A\&A, 415, 499, GR04

Gebhardt, K., Lauer, T. R., Pinkney, J., et al. 2007, 671, 1321, GE07

Goudfrooij P., Schweizer, F., Gilmore, D., \& Whitmore, B. C. 2007, AJ, 133, 2737

Harris, W. E., Kavelaars, J. J., Hanes, D. A., Pritchet, C. J., \& Baum, W. A. 2009, AJ, 137, 3314

Harris W. E., \& Racine, R. 1979, ARA\&A, 17, 241

Hopkins P. F., Cox, T. J., Dutta, S. N., et al. 2009, ApJS, 181, 135

Illingworth G. 1976, ApJ, 204, 73

Kissler M., Richtler, T., Held, E. V., et al. 1994, A\&A, 287, 463, KR94

Lauer T. R., Gebhardt K., Faber S. M., et al. 2007, ApJ, 664, 226

Kormendy J., \& Bender, R. 2009, ApJ, 691, L142, KB09

Lauer T. R. \& Kormendy, J. J. 1986, ApJ Lett., 303, 1

Lee, M. G., Park, H. S., Kim, E., et al. 2008, ApJ, 682, 135

Magorrian J., Tremaine, S., Richstone, D., et al. 1998, AJ, 115, 2285, M98

McLaughlin D. E. 1995, AJ, 109, 2034

Muzzio J. C. 1987, PASP, 99, 245

Ostriker J. P. 1988, in The Harlow Shapley Symposium on Globular Cluster Systems in Galaxies (Dordrecht: Kluwer Academic Publishers), IAU Symp., 126,271

Ostriker J. P., Binney, J., \& Saha, P. 1989, MNRAS, 241, 849

Peng, E. W., Ford, H. C., \& Freeman, K. C. 2004, ApJ, 602, 705

Prugniel P., \& Simien F. 1996, A\&A, 309, 749

Rhode, K. L., \& Zepf, S. E. 2001 AJ, 121 , 210

Rhode, K. L., \& Zepf, S. E. 2003, AJ, 126, 2307

Rhode K. L., \& Zepf, S. E. 2004, AJ, 127, 302, RZ04

Rhode, K. L., Katherine, L., Zepf, S. E., Kundu, A., \& Larner, A. N. 2007, AJ, 134,1403

Richstone D., Ajhar, E. A., Bender, R., et al. 1998, Nature, 395, A14, R98

Schwarzschild, M. 1979, ApJ, 232, 236

Sikkema, G., Peletier, R. F., D.Carter, Valentijn, E. A., \& Balcells, M. 2006, A\&A, 458, 53

Smith B. F., \& Miller, R. H. 1982, Ap. J. 257, 103

Spitler, L. R., Forbes, D. A., Strader, J., Brodie, J. P., \& Gallagher, J. S. 2008, MNRAS, 385, 361

Spitler L. R., Larsen, S. S., Strader, J., et al. 2006, AJ, 132, 1593

Spolaor Ma., Forbes D. A., Hau G. K. T., Proctor R. N., \& Brough S. 2008, MNRAS, 385, 667

Tremaine, S., Gebhardt, K., Bender, R., et al. 2002, ApJ, 574, 740, TR02

Tremaine S. D., Ostriker, J. P., \& Spitzer, L., Jr. 1975, ApJ, 196, 407

Trujillo I., Erwin, P., Asensio Ramos, A., \& Graham, A. W. 2004, AJ, 127, 1917

Van der Marel, R. P. 1999, ApJ, 117, 744, VM99

Verdoes G., Van der Marel, R. P., Carollo, C. M., \& de Zeeuw, P. T. 2000, AJ, 120, 1221, VV00

Vietri M., \& Schwarzschild, M. 1983, ApJ, 269, 487

Zhang Z., Xu, H., Wang, Y., et al. 2007, ApJ, 656, 805, Z07 